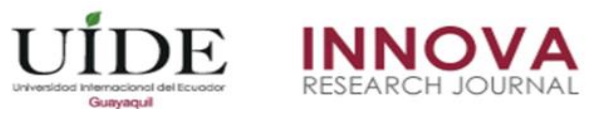

INNOVA Research Journal, ISSN 2477-9024

(Agosto, 2017). Vol. 2, No.8 pp. 155-171

DOI: https://doi.org/10.33890/innova.v2.n8.2017.262

URL: http://revistas.uide.edu.ec/index.php/innova/index

Correo: innova@uide.edu.ec

\title{
Análisis situacional de la formación del piloto y copiloto de la modalidad rally sobre tierra en el Ecuador 2016-2017
}

\section{Situational analysis of the training of the pilot and co-pilot of the modality rally on earth in Ecuador 2016-2017}

Ing. Edgar Gustavo Vera Puebla

Lic. David Job Morales Neira MSc

Ing. Oscar Villavicencio Salazar

Universidad Internacional del Ecuador, Ecuador

$\mathrm{PhD}$. Giceya de la Caridad Maqueira Caraballo

Universidad de Guayaquil, Ecuador

Lic. Roberto Andrés Rojas Bajaña

Unidad Educativa de Fuerzas Armadas Liceo Naval de Guayaquil "Cmdte. Rafael Andrade

Lalama”, Ecuador

Autor para correspondencia: edverapu@internacional.edu.ec, damoralesne@internacional.edu.ec, osvillavicenciosa@internacional.edu.ec,giceyamc@gmail.com,jimurarobert27@hotmail.com Fecha de recepción: 02 de Agosto de 2017 - Fecha de aceptación: 10 de Agosto de 2017

Resumen: En el presente estudio se basa en la preparación física del Piloto-Copiloto del automovilismo en el Ecuador, uno de los problemas es la aplicación de una metodología ajustada a las necesidades de una preparación física, psicológica y fisiológica de forma específica. Se han evidenciado prácticas empíricas, intuitivas, espontaneas, sin respaldo académico que sustentara los métodos de entrenamiento utilizados. En la actualidad favorablemente se han dado extraordinarios avances para mejorar el rendimiento físico y mental. Pilotos ecuatorianos de la era de los 70 afirman que su preparación dependía del mantenimiento exhaustivo del motor y estética del auto, pero no en las estrategias de conducción por la escasa experiencia que tenian en esos momentos como también en la preparación física. El presente artículo presenta dos enfoques (cuantitativo y cualitativo) donde la investigación científica durante el proceso de estudio pretende fortalecer el conocimiento en el área automotriz-deportiva mediante estudios de carácter de campo, bibliográfico y documental. Los principales resultados de las encuestas muestran un alto conocimiento en el sistema y mecanismos del vehículo durante la competición, así mismo los encuestados aseguran que es fundamental la preparación biopsicosocial y cultural del piloto-copiloto en nuestro medio como también los factores que intervienen en la dinámica del automóvil en la competición y finalmente es fundamental la preparación del equipo de trabajo como son la colaboración y cooperación como competencia actitudinal que dependerá el éxito de la carrera. En la entrevista aseguran los participantes que resulta imprescindible la preparación física del piloto-copiloto ya que las pruebas desgastan enormemente al organismo y manifiestan que por sus propios medios entrenan de manera empírica. Cabe recalcar que esta investigación, es inédita en el Ecuador, pero que en otros países ya lo realiza. Finalmente aspiramos que en el Ecuador se considere de una manera formal, programada y preventiva el desarrollo de potencialidades motrices inherentes a la formación del piloto y copiloto para Rally de Tierra.

Palabras claves: rally; piloto -copiloto; hoja de ruta; eficiencia motriz; entrenamiento biopsicofísico 


\begin{abstract}
The present study is based on the physical preparation of the pilots and co-pilots of motor racing in Ecuador; one of the problems is the application of an appropriated methodology adjusted to the needs of a specific physical, psychological and physiological preparation. Empirical, intuitive, spontaneous, non-academic practices have been used to support the training methods. Nowadays, advances have been made to improve mental and physical performance. Ecuadorian pilots of the 70's say that their preparation depended on the exhaustive maintenance of the engine and aesthetics of the car, but not on the strategies of driving because of the limited experience they had at that time as well as in physical preparation. The present article presents two approaches (quantitative and qualitative) where scientific research during the study process aims to strengthen knowledge in the automotivesports area through a field, bibliographic and documentary studies. The main results of the surveys show a high knowledge in the system and mechanisms of the vehicle during the competition, also the respondents assert that the biopsychosocial and cultural preparation of the pilot-copilot in our environment is essential as well as the factors that intervene in the dynamics Of the automobile in the competition and finally it is fundamental the preparation of the work team as they are the collaboration and cooperation as attitudinal competition that will depend on the success of the race. In the interview the participants say that it is essential to prepare the pilot-co-pilot physically because the racing consume a lot of energy of the body and they say that they train by theirselves following an empirical way of training. It should be emphasized that this research is unpublished in Ecuador, but in other countries it is already applied. Finally, we hope that in Ecuador, it will be considered in a formal, programmed and preventive way the development of the driving potential inherent to the physically formation of the pilot and co-pilot for rally car rasing.
\end{abstract}

Key words: rally; pilot, co-pilot; roadmap; motor efficiency; bio-psychophysical training

\title{
Introducción
}

"Un piloto sano, es conocedor de las bondades de la práctica deportiva; transmite valores, la comunidad lo ve como un ejemplo. No sólo es talento para la conducción debe ser partícipe de un programa regular a largo plazo para su evolución racional y motriz”.'(Sainz, 2003, pag. 8).

La Preparación Física del Piloto-Copiloto de automovilismo: uno de los problemas no desarrollados hasta hace poco tiempo por el piloto-copiloto es el déficit de una metodología ajustada a las necesidades de una preparación física, psicológica y fisiológica específica. De manera histórica se han realizado prácticas empíricas, intuitivas, espontaneas, sin respaldo académico que sustentara los métodos de entrenamiento utilizados. En la actualidad favorablemente se han dado extraordinarios avances para la mejora del rendimiento físico y mental (Cohen, 2003, pág. 7).

Para Cohen (2003) en el libro "Manual de entrenamiento para el piloto de automovilismo" establece que en la programación del deportista (piloto-copiloto) no hay una resultados concluyentes, sino que se basan en la adaptación del deportista considerando las características de recepción de la carga física para la competición y post competición. La programación de los deportistas no debe verse como un factor aislado, sino la armonía que debe existir en los aspectos-componentes del entrenamiento deportivo.

La finalidad no es tener un hombre "biomáquina", sino brindar un ambiente de seguridad, confianza y disfrute, instando la eficiencia del entrenamiento en el mínimo tiempo (tiempo 
productivo-tiempo de calidad) de sacrificio y utlidad para el mejoramiento como piloto-copiloto y ser humano.

Según el diario El Comercio (2011) a partir de 1970 se presenta el auge económico (Ecuador se convirtió en nación petrolera), el sector automotriz creció considerablemente. Como hecho histórico se acondicionaban las principales calles de las ciudades de: Guayaquil, Cuenca, Ambato y Riobamba que permitieron dar origen a las competencias de ruta y las de circuito. En la década del 70, aumentó el interés por la cultura de motores en parte por los éxitos de los finlandeses Timmo Makkinen y Hannu Mikola.

Los pilotos Guillermo Ortega, Fausto Merello y Lothar Ranft (alemán radicado en el país), dirigieron el Porsche 908-2 en la 24 Horas de Le Mans (carrera que se realiza todos los años en el mes de junio en el circuito de Sarthe-Francia) organizada por el "Autobmobile Club de I'Ouest (ACO)", puntuable para el campeonato Mundial de Resistencia entre las décadas de 1950 y 1990. En junio de 1974 quedaron séptimos en la general y sexto en la clase de sport-prototipos como el mayor logro en el deporte tuerca conseguido por pilotos nacionales a nivel internacional.

A nivel nacional resalta la carrera "12 Horas Marlboro" realizada en Yahuarcocha ubicada en la provincia de Imbabura, con un recorrido de $10 \mathrm{~km}$. Entre las décadas de 1950 y 1960 se dieron a nivel de Rally las tres primeras vueltas al país. En el año de 1972 se desarrolló la quinta "Vuelta al Ecuador" con recorrido de provincias de la sierra y costa. En un BMW Alpina de $1600 \mathrm{~cm} 3$, el vencedor fue Fernando Madera (piloto ibarreño referente local de la época de oro del automovilismo ecuatoriano), también se encontraron competidores extranjeros: uno de Chile y otro de Perú. Se diseñaron categorías de monoplazas con la finalidad de encontrar talentos que puedan intervenir en competencias nacionales y del extranjero, además se organizaron circuitos trascendentales como la "Copa Caney en Yahuarcocha en 1987" con la victoria de los peruanos Gaspare y Carlos Ibarcenas en un Mazda RX-7.

Fernando Madera es uno de los pilotos más exitosos del automovilismo ecuatoriano hasta 1977 en su año de retiro (Diario El Comercio, 2011). Se inició en los sesenta en el "Club de Automovilismo y Turismo Imbabura (Cati)". Establece que parte de su éxito se debe a la preparación de autos aunque no tenía mayor experiencia conduciendo en el automovilismo. Indica que muy pocas veces se quedó con un auto dañado y cuidó también de la estética de su auto.

Entre los autos que tuvo en su trayectoria están: 1971 el Porsche Carrera 6, BMW 1600 Alpina (utilizó en competencias de rally), con ambos autos ganó diferentes competencias en 1972 quedando campeón nacional, campeón del Rally “Caminos del Inca-Perú". Fernando Madera nació en Ibarra el 2 de marzo de 1941, junto con un grupo de motivados ibarreños promovieron la construcción del autódromo de Yahuarcocha. En 1972 ganó en todas las carreras que intervino, incluyendo la "Vuelta la República coronándose campeón nacional”. En 1977 se retira por motivos de novedades-incoformidades en el reglamento del automovilismo y diferencias con los dirigentes.

\section{Desarrollo}




\section{Objetivos:}

- Analizar la formación del piloto y copiloto de la modalidad Rally sobre tierra en el Ecuador.

- Profundizar en los fundamentos teóricos, científicos e históricos sobre la preparación física, técnica, táctica y psicológica del piloto y copiloto.

- Determinar por medio de las encuestas y entrevistas a los expertos datos reales y precisos que aporten científicamente al presente análisis.

Para tener éxito cómo deportista debemos descubrir a través de la necesidad: $<<l o$ haré $\rangle>,\langle<$ puedo hacerlo $\rangle>, \quad\langle<$ quiero hacerlo $\rangle>$, de una forma total de compromiso. En el momento de planificar debe tomarse en cuenta al deportista como una unidad indivisible, en la cual todos los actos están íntimamente ligados en relacion al ser. No se puede trabajar un aspecto del entrenamiento sin descuidar los demás.

Según Cohen el entrenamiento biopsicofísico está constituido por:

- Aptitud física: corresponde a las capacidades condicionales de fuerza, velocidad, resistencia y flexibilidad. La manifestación de los resultados del "aptitud fisiológica": faculta la efectividad orgánica de las capacidades o valencias físicas correspondientes a la diversidad del gesto motor en los principales aparatos y sistemas como el cardiorrespiratorio, muscular, óseo, neuromuescular, entre otros.

- Aptitud psicológica: se vincula con la voluntad, el espíritu de sacrificio, la inteligencia emocional (interpersonal e intrapersonal), la motivación intrínseca, la tolerancia, el respeto, capacidad de sufrimiento, en definitiva todas aquellas necesarias para llegar a un nivel ulterior corresponden a la composición genética.

En el deporte tuerca, el movimiento deportivo se origina por medio de la percepción sensorial, la vista que a través de la comunicación nerviosa llega al músculo esquelético produciendo la acción motriz. El piloto-copiloto deberá llevar-cumplir con un programa que garantice la automatización efectiva de los movimientos (economizar energía, ganar tiempo de reacción y ejecución).

Para la implementación de un programa de entrenamiento se deberá considerar las cualidades del movimiento: cualitativas y cuantitativas. Las características cualitativas se identifican con el volumen del movimiento (series -repeticiones), acoplamiento, ritmo y fluidez. Las carácterísticas cuantitativas se encuentran las dinámicas y cinemáticas. Las características cinemáticas: tiempo, lugar, espacio, curvas, ángulos, tiempos, aceleraciones, mientras las características dinámicas: fuerza, movimientos angulares, impulso de la fuerza y la proporción del movimiento; el gesto motor es parte de una estructura compleja.

"En el deporte de competición rara vez se produce un milagro"; los logros se dan a través de un modelo de gestión sistematizado, sensibilizado con pares académicos y especialistas 
que suman a un mismo fin. La apertura a la investigación científica, ciencias a fin a la educación, a la actividad física, al deporte y demás que han permitido romper constantemente las marcas. Las condiciones extremas de la competición amerita la perfección de los gestos adquiridos durante un proceso de formación.

El piloto-copiloto debe tener una actitud tenaz, querer ser exitoso, tomarlo como un desafío, que se puede llegar a serlo satisfactoriamente. La puesta en práctica de un programa de entrenamiento biopsicofísico: reforzará de manera significativa la ejecución óptima y equilibrada en el piloto para el manejo en cualquier situación. Aplicando los tipos de evaluación: de entrada (inicial) personalizada por pruebas médicas, test de pruebas de salud (Martínez, Maestría en Educación Física, 2016), test virtual (juego DIRT RALLY), pruebas físicas, pruebas de conocimiento sobre reglamentación, teoría aplicada al contexto de Rally, prueba de simulación de circuitos de competencias en condiciones semejantes: horario, temperatura ambiente, pista, terreno- textura, neumáticos; etapa correspondiente a la fase precompetitiva y competitiva del programa.

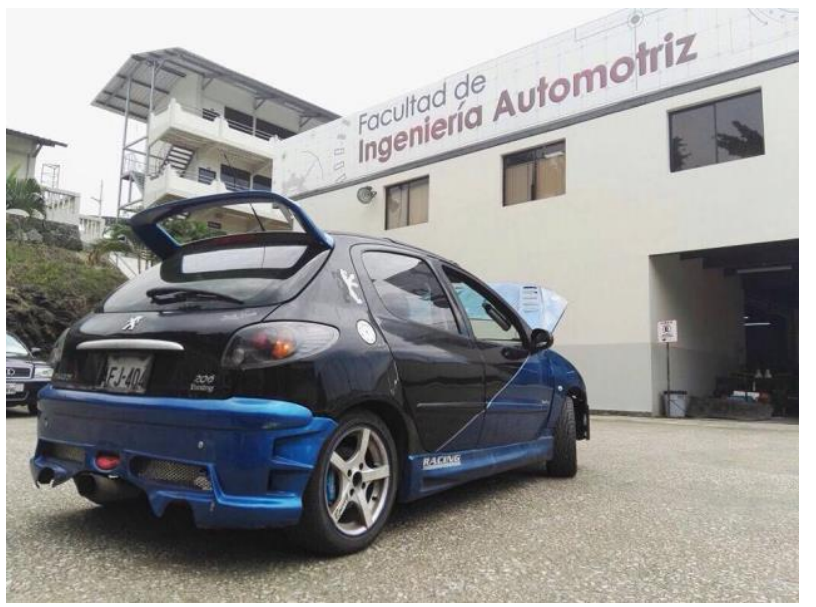

Ilustración 1 Exhibición de autos de Rally en la UIDE-FIA abril 2017

La voluntad es un carácter indispensable para hacer frente a las dificultades, donde no depende de impulsos veloces, sino de una voluntad permanente. El automovilismo es considerado un "Deporte Espectáculo", existe gran influencia de aspectos económicos, sociales, políticos, definiéndose el piloto como un modelo a seguir, siendo escuchado y seguido por los diferentes medios de comunicación. Pihkala en 1930 (finlandés) presenta bosquejos modernos sobre la <<periodización del entrenamiento deportivo〉>. En el automovilismo se busca perfeccionar y acelerar el gesto motriz permitiendo un mejor resultado. 


\section{El rendimiento del entrenamiento}

\begin{tabular}{|c|c|c|c|}
\hline Aspectos sociales & $\begin{array}{c}\text { Aspectos } \\
\text { psicológicos }\end{array}$ & Aspectos técnicos & Eficiencia Física \\
\hline $\begin{array}{ll}\text { - } & \text { Familia } \\
\text { - } & \text { Amigos } \\
\text { - } & \text { Nivel Cultural } \\
\text { - } & \text { Situación } \\
& \text { socioeconómica }\end{array}$ & $\begin{array}{ll}\text { - } & \text { Capacidad } \\
\text { de } & \\
\text { rendimiento } \\
\text { - }\end{array}$ & $\begin{array}{l}\text { - } \begin{array}{l}\text { Destreza de } \\
\text { conducción }\end{array} \\
\text { - Pensamiento } \\
\text { táctico } \\
\text { - Experiencia } \\
\text { en la } \\
\text { competición }\end{array}$ & $\begin{array}{ll}\text { - } & \text { Cordinación } \\
\text { neuromuscular } \\
\text { - } & \text { Inteligencia } \\
\text { motora } \\
\text { - } \\
\text { Aptitudes } \\
\text { físicas } \\
\text { - Aptitudes } \\
\text { físiológicas }\end{array}$ \\
\hline
\end{tabular}

Ilustración 2 El rendimiento del entrenamiento

Fuente: (Cohen, Manual de entrenamiento para el piloto de automovilismo, 2016, pág. 28)

El programa de entrenamiento biopsicofísico partirá de una base de:

- Preparación Física General, con la finalidad de mejorar el <<rendimiento fisiológico〉> que suministre una cimiento sólido y equilibrado.

- Preparación Física Específica, se definirán actividades para los músculos específicos que participan en el gesto motor.

Según Cohen el trabajo físico se basará sobre los siguientes principios fisiológicos:

\section{Tabla 1 Principios fisiológicos}

\begin{tabular}{ll}
\hline \multicolumn{1}{c}{ Principios generales } & \multicolumn{1}{c}{ Principios específicos } \\
\hline -Individualidad de la carga & -Multirateralidad \\
-Adaptación a la intensidad & -Especificidad \\
-Sobrecarga & -Estimulación voluntaria \\
-Continuidad (frecuencia) & -Transferencia \\
-Progresión & -Eficacia \\
\hline
\end{tabular}

Fuente: (Cohen, Manual de entrenamiento para el piloto de automovilismo, 2016, pág. 30) 


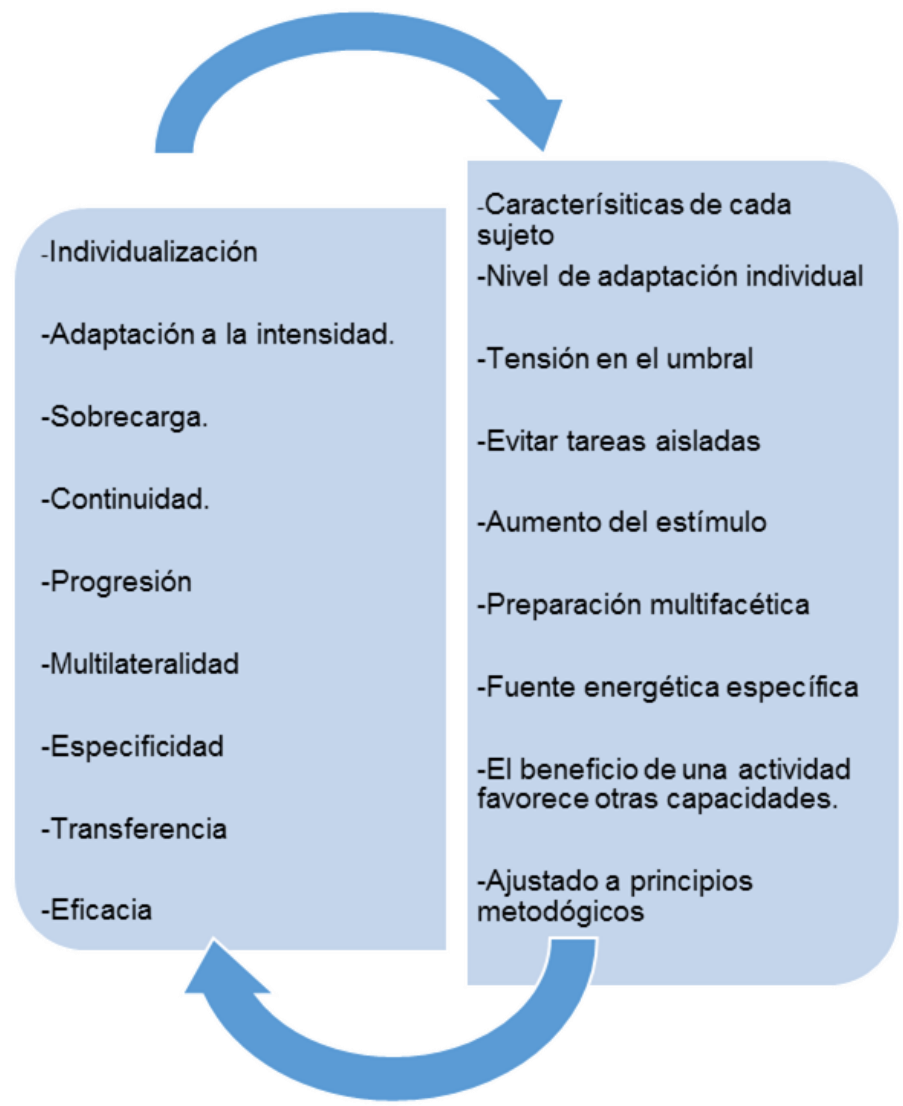

Ilustración 3 Características de cada principio fisiológico

Fuente: (Cohen, Manual de entrenamiento para el piloto de automovilismo, 2016, pág. 31-32)

Elaborado por: Morales Job, Vera Edgar.

La eficiencia física, entrenamiento de la fuerza y cambios neuronales: dependen del óptimo estímulo nervioso positivo.

Tabla 2 Mejora de la eficiencia física (Cohen, 2016)

\begin{tabular}{|c|c|c|}
\hline \multicolumn{3}{|c|}{ Mejora de la Eficiencia Física por Cohen (2016)-Entrenamientos tipos de fuerza } \\
\hline $\begin{array}{l}\text { Aptitutd } \\
\text { Física }\end{array}$ & Objetivo & Sistemas y Métodos \\
\hline $\begin{array}{l}\text { Fuerza } \\
\text { máxima }\end{array}$ & $\begin{array}{l}\text { Hipertrofia muscular, mejora de la } \\
\text { Coordinación neuromuscular }\end{array}$ & $\begin{array}{l}\text {-Cargas piramidales, } \\
\text {-Método de pesas, } \\
\text {-Grandes aparatos como barras y } \\
\text { paralelas. } \\
\text {-Pocas repeticiones } \\
\text {-Cargas máximas y submáximas. }\end{array}$ \\
\hline $\begin{array}{c}\text { Fuerza } \\
\text { velocidad }\end{array}$ & $\begin{array}{c}\text { Mejora de la coordinación intramuscular e } \\
\text { intermuscular }\end{array}$ & $\begin{array}{l}\text {-Ejericicios-autocargas. } \\
\text {-Resistencias medias o bajas. } \\
\text {-Alta velocidad de ejecución. }\end{array}$ \\
\hline & Realizar mayor número de repeticiones & $\begin{array}{l}\text {-Autocargas (propio peso). } \\
\text {-Balones medicinales }\end{array}$ \\
\hline Fuerza & & -Pesos. \\
\hline resistencia & & -Mancuernas \\
\hline & & $\begin{array}{l}\text {-Resistencia media permita mayor } \\
\text { cantidad de repeticiones. }\end{array}$ \\
\hline
\end{tabular}


Tabla 3 Mejora de la eficiencia física (Cohen, 2016)

\begin{tabular}{|c|c|c|}
\hline \multicolumn{3}{|c|}{ Mejora de la Eficiencia Física por Cohen (2016)-Entrenamientos tipos de Resistencia } \\
\hline $\begin{array}{l}\text { Aptitud } \\
\text { Física }\end{array}$ & Objetivo & Sistemas y Métodos \\
\hline $\begin{array}{l}\text { Resistencia } \\
\text { General }\end{array}$ & $\begin{array}{l}\text { Mejora de la oxigenación celular y la capilarización del } \\
\text { sistema muscular esquelético; mayor volumen de sangre } \\
\text { impulsada, ahorro de consumo de oxígeno cardiáco. }\end{array}$ & $\begin{array}{l}\text {-Carrera continua. } \\
\text {-Fartlek. } \\
\text {-Natación frecuencia cardiaca } \\
\text { entre } 140-160 \text { ppm. } \\
\text {-Circuito de resistencia con } \\
\text { cargas de baja y media intensidad } \\
\text { entre } 15 \text {-20 repeticiones. }\end{array}$ \\
\hline $\begin{array}{l}\text { Resistencia } \\
\text { Específica }\end{array}$ & $\begin{array}{c}\text { Mantenimiento del nivel de velocidad, fuerza o técnica } \\
\text { motriz, consecuencia de la tolerencia a la fatiga a pesar } \\
\text { del aumento en la intensidad de cargas; tolerancia al } \\
\text { lactato, adecuada eliminación de sustratos y residuos } \\
\text { metabólicos. }\end{array}$ & $\begin{array}{l}\text {-Entrenamiento intervalos con } \\
\text { pausas incompeltas. } \\
\text {-Métodos de repeticiones con } \\
\text { cargas de intensidad media-alta. } \\
\text {-Circuito de entrenamiento } \\
\text { anaeróbico con cargas medias altas } \\
\text { entre } 10-15 \text { repeticiones y } \\
\text { recuperación entre ejercicios. }\end{array}$ \\
\hline
\end{tabular}

Por otro lado Martínez (2016) plantea "Pruebas de condición Física para la salud UAM (BAFS)" para determinar la resistencia: "Test de $2 \mathrm{Km}$ caminando"; velocidad: "carrera de 20 metros", fuerza: "sentarse y levantarse" en 30" la mayor cantidad de repeticiones de Rikli y Jones (2001).

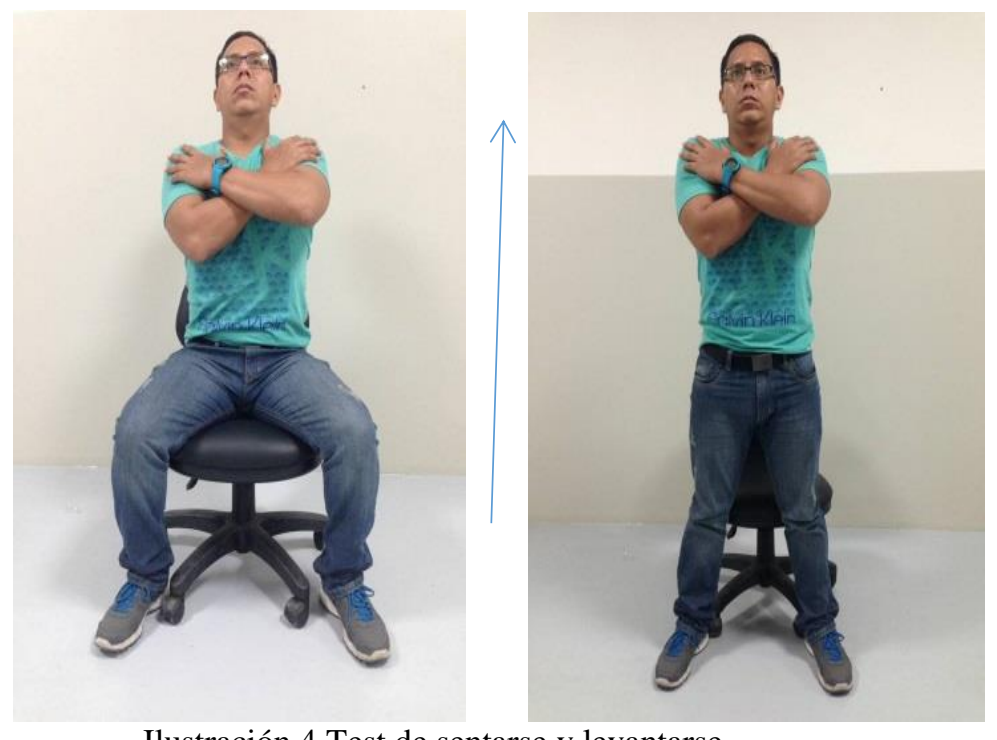

Ilustración 4 Test de sentarse y levantarse

Fuerza extremidades superiores: flexo - extensiones con $1 \mathrm{Kg}$, fuerza de tronco: 
- Dorso: Test de Biering -Sorensen

- para la evaluación de la resistencia de los músculos extensores del tronco (Recio, Barbado, López \& Vera, 2014).

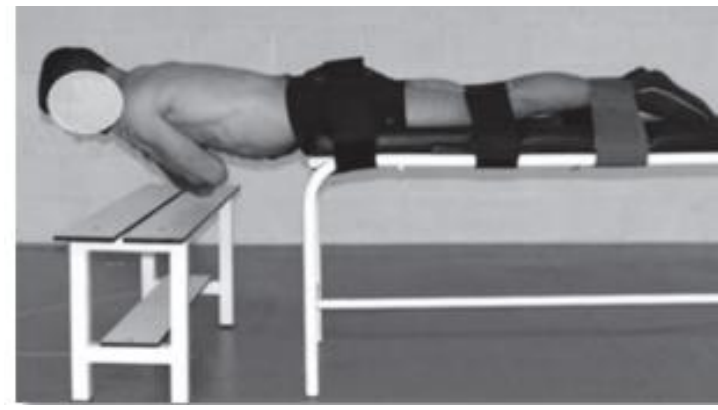

Ilustración 5 Test de Biering -Sorensen

Test de flexibilidad:

-Hombros: Back Scratch (rascarse la espalda) Test de Rikli y Jones

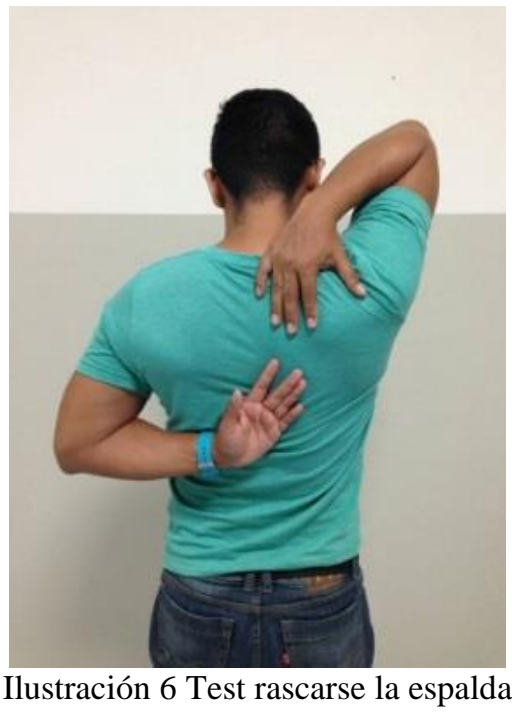

-Cadera: Chair Sit and Reach (sentarse y alcanzar) Test de Rikli y Jones
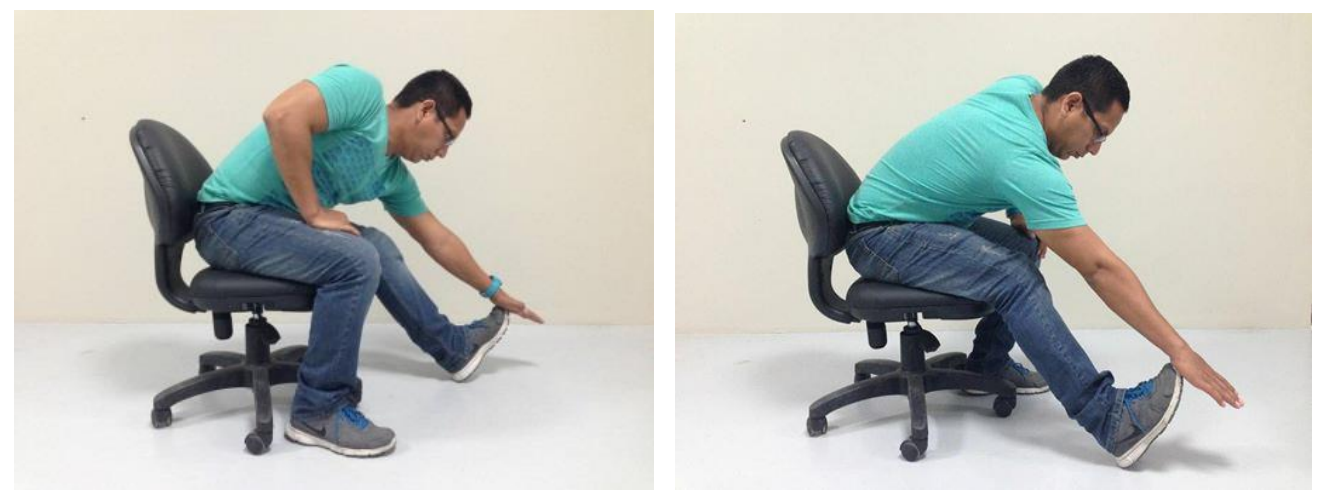
En este sentido Martínez (2016) cita a la Oganización Mundial de la Salud (2006) donde resalta "La salud es el estado de completo bienestar físico, mental y social, y no solamente la ausencia de afecciones o enfermedades". Recomienda efectuar antes de participar en un programa de entrenamiento se aplique una breve encuesta-cuestionario de salud, pruebas médicas y físicas básicas: dinamometría de prensión manual, peso, talla, salto de longitud horizontal con pies juntos, carrera de ida y vuelta 10 x 5, tapping test, flamingo (test de equilibrio monopodal en 60 segundos), abdominales en 30 segundos, Course Navette, índice de masa corporal, salto vertical, entre otros.

\section{Metodología}

El presente artículo se basa en los dos enfoques (cuantitativo y cualitativo) donde la investigación científica durante el transcurso propende fortalecer el conocimiento en el área automotriz-deportiva. Hernández, Fernández \& Baptista (2006) citan a Grinnell (1997) donde resalta la relación entre ambos paradigmas: la observación y valoración de fenómenos. Proponer ideas producto de la observación y evaluación ejecutadas. Identificar el avance de las ideas sobre la base de las pruebas o del análisis. Plantear nuevas observaciones y valoraciones para esclarecer, alterar, y estipular las suposiciones e ideas e incluso concebir otras. Hernández plantea las características que debe cumplir del enfoque cuantitativo. Debe plantearse una pregunta:

¿Se considera a la preparación física como componente en la formación integral del piloto y copiloto de Rally sobre Tierra en el Ecuador?

Parte de un análisis de los hechos más relevantes del Rally en el Ecuador por medio de la investigación efectuada por el Diario el Comercio. La recolección de datos evidencia la medición de la muestra; encuesta aplicada a pilotos, copilotos de rally sobre tierra en actividad deportiva, docentes y estudiantes de la Universidad Internacional del Ecuador-Sede Guayaquil, Facultad de Ingeniería Automotriz.

El objetivo del cuestionario es identificar los componentes inherentes a la formación del piloto y copiloto nacional de Rally sobre Tierra. El cuestionario se encuentra dirigido a deportistas: pilotos- copilotos activos de Rally, directivos, dirigentes deportivos y docentes de la Facultad de Ingeniería Automotriz de la Universidad Internacional del Ecuador, sede Guayaquil. Este instrumento está basado en los Masters en Educación Física Palomar (2014-2015) y Sanz (2012-2013).

Para ello, al lado de cada frase se encuentra una escala de 1 a 4, valor que corresponde: el 4 al valor cualitativo "totalmente de acuerdo", 3 "de acuerdo", 2 "desacuerdo" y 1 "totalmente en desacuerdo".

Tabla 4 Valor cualitativo de Palomar y Sanz (2012-2014)

1
2
Totalmente desacuerdo
Desacuerdo




\begin{tabular}{cc}
\hline 3 & De acuerdo \\
4 & Totalmente de acuerdo \\
\hline
\end{tabular}

Tabla 5 Datos de los encuestados

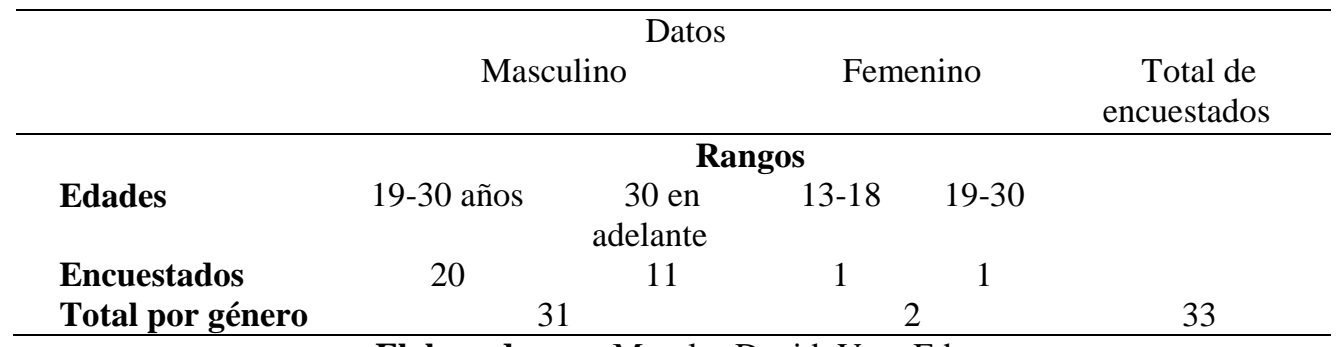

Elaborado por: Morales David, Vera Edgar

Tabla 6 Porcentaje de encuestados por género

\begin{tabular}{ccc}
\hline Masculino & Femenino & Total \\
\hline 31 & 2 & 33 \\
$93,94 \%$ & $6,06 \%$ & $100,00 \%$
\end{tabular}

Elaborado por: Morales David, Vera Edgar \& Roberto Rojas

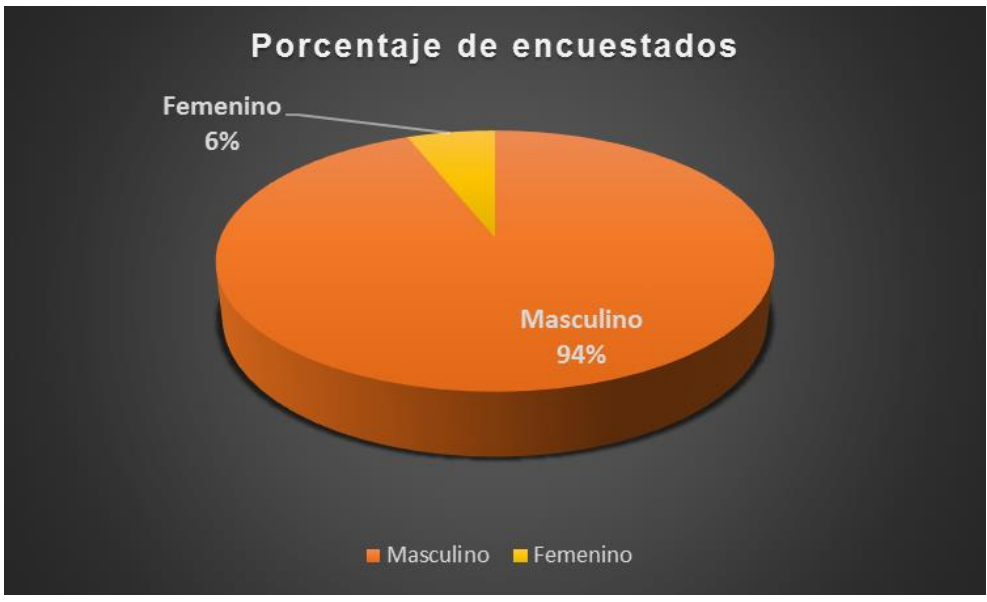

Ilustración 8 Gráfico del porcentaje de encuestados

Tabla 7 Estadística descriptive

\begin{tabular}{lc}
\hline \multicolumn{2}{c}{ Análisis descriptivo } \\
\hline & \\
Media & 31,45454545 \\
Error típico & 1,486104138 \\
Mediana & 30 \\
Moda & 30 \\
Desviación estándar & 8,537018321 \\
\hline
\end{tabular}




\begin{tabular}{lc}
\hline Varianza de la muestra & 72,88068182 \\
Curtosis & 0,351042687 \\
Coeficiente de asimetría & 0,800057596 \\
Rango & 35 \\
Mínimo & 18 \\
Máximo & 53 \\
Suma & 1038 \\
Cuenta & 33 \\
Mayor (1) & 53 \\
Menor(1) & 18 \\
Nivel de confianza (95,0\%) & 3,027095071 \\
\cline { 2 - 2 } Elaborado Roberto Rojas Bajaña, Morales David \& Vera Edgar
\end{tabular}

\section{Resultados}

Tabla 8 Resultados generales de cada encuesta

\begin{tabular}{cccccc}
\hline Ítem & $\begin{array}{c}1 \\
\text { Totalmente en desacuerdo }\end{array}$ & $\begin{array}{c}2 \\
\text { Desacuerdo }\end{array}$ & $\begin{array}{c}3 \\
\text { De acuerdo }\end{array}$ & $\begin{array}{c}4 \\
\text { Totalmente de acuerdo }\end{array}$ & Total \\
\hline $\mathbf{2}$ & $3,03 \%$ & $0,00 \%$ & $3,03 \%$ & $93,94 \%$ & $100,00 \%$ \\
$\mathbf{3}$ & $3,03 \%$ & $18,18 \%$ & $27,27 \%$ & $51,52 \%$ & $100,00 \%$ \\
$\mathbf{4}$ & $3,03 \%$ & $6,06 \%$ & $33,33 \%$ & $57,58 \%$ & $100,00 \%$ \\
$\mathbf{5}$ & $3,03 \%$ & $0,00 \%$ & $48,48 \%$ & $48,48 \%$ & $100,00 \%$ \\
$\mathbf{6}$ & $12,12 \%$ & $27,27 \%$ & $30,30 \%$ & $30,30 \%$ & $100,00 \%$ \\
$\mathbf{7}$ & $0,00 \%$ & $0,00 \%$ & $18,18 \%$ & $81,82 \%$ & $100,00 \%$ \\
$\mathbf{8}$ & $0,00 \%$ & $3,03 \%$ & $36,36 \%$ & $60,61 \%$ & $100,00 \%$ \\
$\mathbf{9}$ & $0,00 \%$ & $0,00 \%$ & $36,36 \%$ & $63,64 \%$ & $100,00 \%$ \\
$\mathbf{1 0}$ & $0,00 \%$ & $0,00 \%$ & $0,00 \%$ & $100,00 \%$ & $100,00 \%$ \\
$\mathbf{1 1}$ & $0,00 \%$ & $3,03 \%$ & $15,15 \%$ & $81,82 \%$ & $100,00 \%$ \\
$\mathbf{1 2}$ & $0,00 \%$ & $0,00 \%$ & $27,27 \%$ & $72,73 \%$ & $100,00 \%$ \\
$\mathbf{1 3}$ & $0,00 \%$ & $0,00 \%$ & $33,33 \%$ & $66,67 \%$ & $100,00 \%$ \\
$\mathbf{1 4}$ & $0,00 \%$ & $0,00 \%$ & $42,42 \%$ & $57,58 \%$ & $100,00 \%$ \\
$\mathbf{1 5}$ & $0,00 \%$ & $3,03 \%$ & $24,24 \%$ & $72,73 \%$ & $100,00 \%$ \\
$\mathbf{1 6}$ & $0,00 \%$ & $0,00 \%$ & $33,33 \%$ & $66,67 \%$ & $100,00 \%$ \\
\hline
\end{tabular}

Elaborado por: Morales David, Vera Edgar

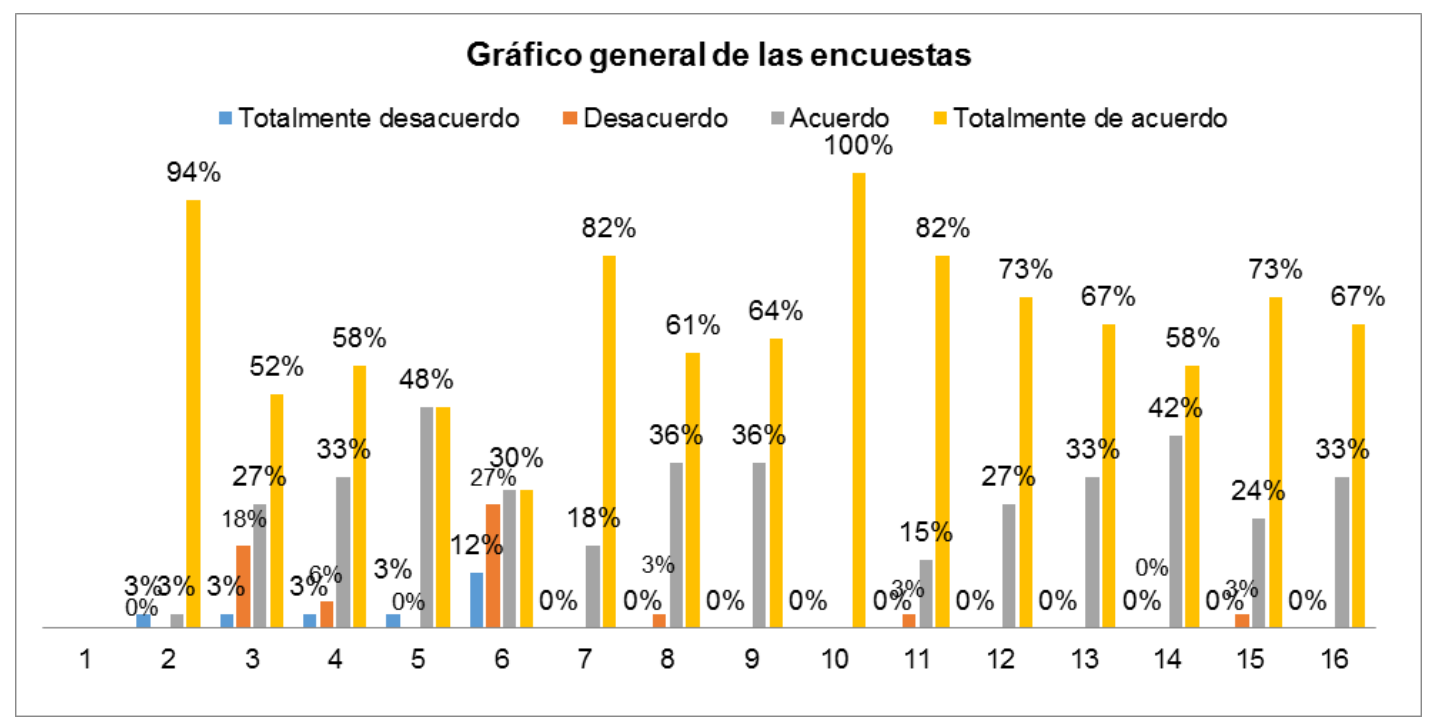


Ilustración 9 Gráfico de los resultados generales de las encuestas

Elaborado por: Morales David, Vera Edgar

Los principales resultados y su análisis se lo detalla de la siguiente forma:

A continuación se van a analizar los ítems de acuerdo a los aspectos del entrenamiento deportivo según Dietrich Harre (1987) en la publicación Teoría del entrenamiento deportivo y destrezas cognitivas, procedimentales y actitudinales :

\section{Competencia cognitiva}

- Según la pregunta $\mathrm{N}^{\circ} 7$ "El diseño, elaboración y socialización de una hoja de ruta permite minimizar el tiempo de recorrido en una competencia deportiva”. El $0 \%$ está totalmente desacuerdo, el $0 \%$ en desacuerdo, el 18, $18 \%$ de acuerdo y el 81, $82 \%$ totalmente de acuerdo.

- Según la pregunta $\mathrm{N}^{\circ} 10$ "El piloto y el copiloto deben dominar la reglamentación de competencia deportiva vigente independiente la modalidad a intervenir". El $0 \%$ está totalmente en desacuerdo, el $0 \%$ en desacuerdo, el $0 \%$ de acuerdo y el $100 \%$ está totalmente de acuerdo.

- Según la pregunta $\mathrm{N}^{\circ} 11$ "El piloto y copiloto deben conocer y dominar los principios de funcionamiento de sistemas y mecanismos en un vehículo de competición”. El $0 \%$ está totalmente en desacuerdo, el 3, $03 \%$ en desacuerdo, el 15, $15 \%$ de acuerdo y el 81, 82\% está totalmente de acuerdo.

\section{Competencia procedimental}

- Según la pregunta $\mathrm{N}^{\circ} 2$ indica "La preparación física es un componente importante en la formación del piloto y copiloto para Rally de Tierra”. El 3,03 \% está totalmente en desacuerdo, el $0 \%$ en desacuerdo, el 3,03\% de acuerdo y el 93,94\% totalmente de acuerdo.

- Según la pregunta $N^{\circ} 3$ indica "Para lograr la formación de un piloto y copiloto se requiere un elevado financiamiento económico". El 3,03\% está totalmente desacuerdo, el 18, $18 \%$ está desacuerdo, el 27,27\% de acuerdo y el 51, $52 \%$ están totalmente de acuerdo.

- Según la pregunta $\mathrm{N}^{\circ} 5$ indica "Mejorar la eficiencia física a través del entrenamiento de diferentes tipos de fuerzas logran un acondicionamiento físico idóneo para la competencia”. El 3,03\% totalmente desacuerdo, $0 \%$ desacuerdo, 48, $48 \%$ de acuerdo y el $48,48 \%$ totalmente de acuerdo.

- $\quad$ Según la pregunta $\mathrm{N}^{\circ} 8$ “Es importante un programa de entrenamiento y competición basado en las relaciones de adaptación conforme a los factores medioambientales (altura, calor, frío, zona geográfica y huso horario)". El 0\% está totalmente está en 
desacuerdo, el 3, $03 \%$ en desacuerdo, el 36, $36 \%$ de acuerdo y el 60, $61 \%$ están totalmente de acuerdo.

- Según la pregunta $\mathrm{N}^{\circ} 9$ indica "Es significativo el cumplimiento de las partes del programa de entrenamiento deportivo aplicado a pilotos y copilotos: preparatorio, competitivo y transitorio”. El $0 \%$ está totalmente en desacuerdo, el $0 \%$ en desacuerdo, el $36,36 \%$ de acuerdo y el 63,64\% totalmente de acuerdo.

- Según la pregunta $\mathrm{N}^{\circ} 13$ indica "Es primordial la ejecución de ejercicios de movilidad articular y el entrenamiento de la flexibilidad", el $0 \%$ está totalmente desacuerdo, el $0 \%$ desacuerdo, el 33, 33\% está de acuerdo y el 66, 67\% está totalmente de acuerdo.

\section{Competencia actitudinal}

- Según la pregunta $N^{\circ} 4$ indica "Es relevante el entrenamiento psicológico orientado a la competición". E1 3,03 \% está en totalmente desacuerdo, el 6,06 \% en desacuerdo, el 33,33 $\%$ de acuerdo y el $57,58 \%$ totalmente de acuerdo.

- Según la pregunta $\mathrm{N}^{\circ} 6$ indica "La herencia-genética son determinantes para la constitución de un piloto y copiloto para Rally de Tierra”. El 12,12\% está totalmente de desacuerdo, el 27,27 \% en desacuerdo, el 30, $30 \%$ de acuerdo y el 30, $30 \%$ están totalmente de acuerdo.

- Según la pregunta $\mathrm{N}^{\circ} 12$ indica que "El trabajo en equipo y las relaciones interpersonales es una estrategia fundamental para alcanzar el éxito deportivo”. El $0 \%$ está totalmente desacuerdo, el $0 \%$ en desacuerdo, el 27, $27 \%$ de acuerdo, y el 72, $73 \%$ está totalmente de acuerdo.

- Según la pregunta $\mathrm{N}^{\circ} 14$ indica “Es sustancial para todo el equipo el entrenamiento invisible (entrenamiento que se lleva antes y después del cumplimiento del programa establecido)"..El $0 \%$ está totalmente desacuerdo, el $0 \%$ desacuerdo, el $42 \%$ de acuerdo y el $58 \%$ totalmente de acuerdo.

- Según la pregunta $\mathrm{N}^{\circ} 15$ indica debe cumplirse con "La revisión periódica médica para el piloto y copiloto". El $0 \%$ totalmente desacuerdo, $0 \%$ desacuerdo, el 33, $33 \%$ de acuerdo y el 66,67\% totalmente de acuerdo.

- Según la pregunta $\mathrm{N}^{\circ} 16$ indica "Debe estar presente la revisión periódica médica para el piloto y copiloto". El $0 \%$ totalmente desacuerdo, el $0 \%$ desacuerdo, el 33, $33 \%$ de acuerdo, y el 66, $67 \%$ está totalmente de acuerdo. 


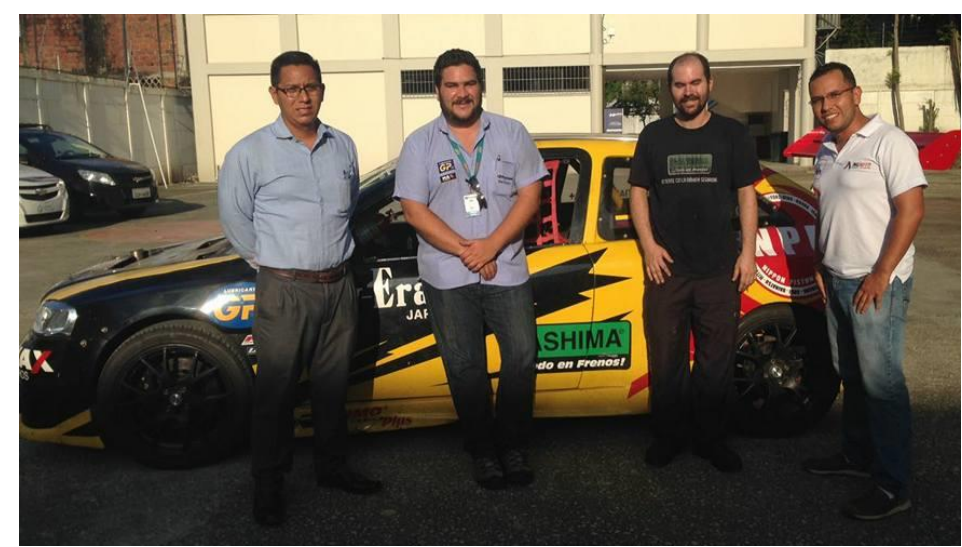

Ilustración 10 Análisis de entrevista a especialistas

Según Ibarra (2017), actual dirigente de la Federación Ecuatoriana de Rally indica que el Ministerio del Deporte del Ecuador, desde el año 2013 propone la toma de pruebas de "aptitud física" con el aval de un médico deportólogo, para evaluar las capacidades físicas de los deportistas de manera previa a la competencia. A su vez, indica que el Ministerio del Deporte en la actualidad no ha formalizado las mismas. Es Ingeniero Automotriz de profesión aplica la preparación del vehículo como parte fundamental. Tuvo la oportunidad de insertarse en el mundo del automovilismo desde los 12 años. En su palmarés como deportista presenta la participación en las carreras "Vuelta a la República del Ecuador" los años 2007, 2010, 2011 y 2014 obteniendo la victoria en la cuarta oportunidad en una categoría intermedia.

Manifiesta que la vuelta a la República tiene un periodo de duración cerca a las 10 horas de competencia. Por tal motivo no sólo es necesario tener en consideración la preparación del auto, sino también darle la debida atención a la formación de carácter científico y potenciar las capacidades físicas del piloto y copiloto para Rally de Tierra del Ecuador. La prueba se realiza en <<caminos de segundo orden>> los cuales desgastan de gran manera al organismo. Para determinar el camino de competencia, se establece de forma previa el levantamiento de la hoja de ruta por lo menos en 5 revisiones. De igual manera Johann Moncayo \& Sebastián Olmos (2017), piloto y copiloto respectivamente, ex alumnos -Ingenieros Automotrices graduados de la UIDE-FIA indican que el Rally es un deporte de alto costo, el cual por general se encuentra financiado por la empresa privada. Expresan que por iniciativa-necesidad deben ajustarse a un programa de entrenamiento biopsicofísico que faculte tolerar las altas exigencias físicas del deporte tuerca.

\section{Discusión}

- Según las preguntas 7, 10 y 11 de la <<competencia cognitiva〉>, el 87,88\% de los encuestados están totalmente de acuerdo en que el piloto y copiloto deben prepararse tácticamente de acuerdo al recorrido, reglamentación y dominar los principios de funcionamiento de sistema y mecanismos del vehículo en la competición.

- Según las preguntas 2,5, 9 y 13 de la <<competencia procedimental >> el 68,18\% de los encuestados están convencidos que el piloto y copiloto se sujeten a programaciones de entrenamiento adecuado y por fases como son: preparatorio, competitivo y transitorio que 
beneficien la condición física. Esto genera el aumento del control motor y la eficiencia física antes, durante y después de la competición; además el piloto y copiloto son seres humanos que dependen del factor biopsicosocial y cultural del Ecuador; mientras, las preguntas 3 y 8 el 56,06\% de los encuestados afirman que para lograr preparar al piloto y copiloto es necesario tener en cuenta los factores climáticos en pista, el tipo de neumático y mantenimiento que necesita el automóvil y eso requiere de un alto porcentaje de requerimiento económico.

- Según las preguntas 12, 14 y 15 de la <<competencia actitudinal >> el 65,80\% de los encuestados consideran que es indispensable y fundamental el trabajo en equipo que comprende las actitudes de cada uno de los integrantes en su punto de trabajo donde premisa la cooperación y colaboración que es un factor positivo para la competición.

\section{Conclusiones}

Se realizó estudios de carácter bibliográfico y documental en cada uno de los documentos que sostienen la preparación física del piloto y copiloto donde se estipuló fundamentos teóricos, científicos e históricos que sustentaron científicamente el presente análisis.

Se determinó por medio de las encuestas que es importante contar con un preparador físico o profesor de EF para la formación del piloto y copiloto correspondiente a aspectos teóricos, tácticos, físicos y psicológicos de acuerdo al recorrido-prueba. En la parte procedimental se debe ponderar la condición aptitud física y actitud psicológica, sin dejar a un lado el uso de la TICprogramas virtuales de entrenamiento generando el aumento del control y la eficiencia motriz: antes, durante y después de la competición, y finalmente premisa la cooperación y colaboración del equipo como un factor relevante para la competición.

\section{Bibliografía}

Casto Recio, David Barbado, Alejandro López \& Francisco Vera. (2014). Test de campo para valorar la resistencia de los músculos del tronco. Obtenido de http://www.raco.cat/index.php/ApuntsEFD/article/viewFile/280885/368549

Cohen, R. (2003). Manual de entrenamiento para el Piloto de automovilismo, planificación y organizacion del entrenamiento. Madrid, España: Librerías deportivas Esteban Sanz.

Cohen, R. (25 de agosto de 2016). Manual de entrenamiento para el piloto de automovilismo. España: Librerias Deportivas Esteban Sanz, S.L.

Diario El Comercio. (11 de diciembre de 2011). El Comercio. Obtenido de http://www.elcomercio.com/deportes/carburando/fernando-madera-historia-referentelocal.html

El Comercio. (11 de noviembre de 2011). El automivilismo en el Ecuador. Obtenido de http://www.elcomercio.com/deportes/carburando/automovilismo-ecuador.html 
El Comercio. (16 de abril de 2013). El Comercio. Obtenido de http://www.elcomercio.com/deportes/daniel-galarza-dio-salto-al.html

Harre, D. (1987). Teoría dle entrenamiento deportivo. La Habana: Stadium SRL.

Johann Moncayo, Sebastián Olmos. (abril de 2017). Entrevista a pilotos y copilotos. Ingenieros graduados de la UIDE . Guayaquil, Guayas, Ecuador: Universidad Internacional del Ecuador, Sede Guayaquil. Facultad de Ingeniería Autmotriz.

Martínez, V. (2016). Maestría en Educación Física. Pruebas de salud. Guayaquil, Guayas, Ecuador: Universidad Autónoma de Madrid.

Martínez, V. (octubre de 2016). Pruebas de condición Física para la salud USM (BAFS). Guayaquil, Guayas, Ecuador: Universidad Autónoma de Madrid.

Oganización Mundial de la Salud. (Octubre de 2006). Constitución de la Organización Mundial de la Salud. Obtenido de http://www.who.int/governance/eb/who_constitution_sp.pdf

Roberto Hernández Sampieri, Carlos Fernández Collado \& Pilar Baptista Lucio. (2006). Metodología de la investigación. Obtenido de https://competenciashg.files.wordpress.com/2012/10/sampieri-et-al-metodologia-de-lainvestigacion-4ta-edicion-sampieri-2006_ocr.pdf 